EESTI NSV TEADUSTE AKADEEMIA TOIMETISED. 28. KOIDE

FUOSIKA * MATEMAATIKA. 1979, NR. 2

ИЗВЕСТИЯ АКАДЕМИИ НАУК ЭСТОНСКОИ ССР. ТОМ 28 ФИЗИКА * МАТЕМАТИКА. 1979, № 2

В. ОЛЬМАН

удк 519.28

\title{
ПРОВЕРКА ГИПОТЕЗЫ О ПРИНАДЛЕЖНОСТИ ПАРАМЕТРА ЛИНЕЙНОИ РЕГРЕССИОННОЙ МОДЕЛИ ЗАДАННОМУ эллипсоиду
}

\author{
(Представлена Н. Алумяэ)
}

Рассмотрим схему наблюдений по регрессионной модели

$$
\mathbf{y}_{i}=\mathbf{x}_{i}^{\mathrm{T}} \theta+\varepsilon_{i}, \quad i=1,2, \ldots, n,
$$

где случайные величины $\varepsilon_{i}, i=1,2, \ldots, n$, независимы и распределены по нормальному закону $N(0,1) ; m$-мерные неслучайные векторы $\mathbf{x}_{1}, \mathbf{x}_{2}, \ldots, \mathbf{x}_{n}$ считаются известными, а $\theta-m$-мерный неизвестный наблюдателю неслучайный вектор, $n>m$.

Во многих работах $\left[{ }^{1-3}\right]$ по оцениванию вектора регрессионной модели (1) предполагается, что неизвестный вектор $\theta$ лежит в фиксированном эллипсоиде. В настоящей статье строятся оптимальные в некотором смысле критерии, позволяющие проверить истинность этого априорного знания.

Для проверки гипотезы $H_{0}:\left(\theta-\theta_{0}\right)^{\mathrm{T}} \mathbf{A}\left(\theta-\theta_{0}\right) \leqslant r^{2}$ против альтернативы $K:\left(\theta-\theta_{0}\right)^{\mathrm{T}} \mathbf{A}\left(\theta-\theta_{0}\right)>r^{2}$, где $m$-мерный вектор $\theta_{0}$ и неотрицательно определенная матрица А порядка $(m \times m)$ считаются известными, будем использовать критические области $W$ вида

$$
W(\mathbf{T}, \mathbf{u})=\left\{\mathbf{Y} \in R^{n}:(\mathbf{T Y}+\mathbf{u})^{\mathrm{T}} \mathbf{A}(\mathbf{T Y}+\mathbf{u}) \geqslant 1\right\},
$$

где $\mathbf{Y}^{\mathrm{T}}=\left(y_{1}, y_{2}, \ldots, y_{n}\right), \mathbf{T}$ и $\mathbf{u}$ неслучайные матрица и вектор порядков $(m \times n)$ и $m$ соответственно. Каждому критерию $W$ поставим в соответствие функцию

$$
\gamma(\mathbf{T}, \mathbf{u}, v)= \begin{cases}\alpha(\mathbf{T}, \mathbf{u}, v)=\sup _{\theta \in S(v)} P(W(\mathbf{T}, \mathbf{u}) \mid \theta), & v \leqslant r, \\ \beta(\mathbf{T}, \mathbf{u}, v)=\inf _{\theta \in S(v)} P(W(\mathbf{T}, \mathbf{u}) \mid \theta), & v>r,\end{cases}
$$

где $S(v)$ - сфера $\left\{\theta \in R^{m}:\left(\theta-\theta_{0}\right)^{\mathrm{T}} \mathbf{A}\left(\theta-\theta_{0}\right)=v^{2}\right\}$, а $P(W(\mathbf{T}, \mathbf{u}) \mid \theta)$ - вероятность попадания вектора $\mathbf{Y}$ в критическую область $W$. Таким образом, с помощью функции $\gamma(\mathbf{T}, \mathbf{u}, v)$ мы произвели факторизацию класса гипотез и класса альтернатив и получили некоторую аналогию задачи проверки гипотезы об одномерном параметре $v$, в которой $\gamma(\mathbf{T}, \mathbf{u}, v)-$ функция мощности критерия $W(\mathbf{T}, \mathbf{u})$.

Введем в рассмотрение множество $N_{\alpha}$, состоящее из критериев $W$ вида (2) таких, что 


$$
\max _{v \leqslant r} \gamma(\mathbf{T}, \mathbf{u}, v)=\alpha,
$$

т: е. $W(\mathbf{T}, \mathbf{u}) \in N_{\alpha}$, если наибольшая ошибка первого рода критерия $W(\mathbf{T}, \mathbf{u})$ равна $\alpha$.

Т еорем а 1. Пусть $\mathbf{A}=\mathbf{X}^{\mathrm{T}} \mathbf{X}$ - положительно определенная матрица, $\mathbf{X}=\left(\mathbf{x}_{1} \mathbf{x}_{2}: \cdots: \mathbf{x}_{n}\right)$. Тогда в классе $N_{\alpha}$ критерий $W_{0}$, которому соответствуют $\mathbf{T}_{0}=t_{0}\left(\mathbf{X}^{\mathrm{T}} \mathbf{X}\right)^{-1} \mathbf{X}^{\mathrm{T}} u \mathbf{u}_{0}=-\mathbf{T}_{0} \mathbf{X} \theta_{0}$, где $t_{0}$ выбирается из условия $\gamma\left(\mathbf{T}_{0}, \mathbf{u}_{0}, r\right)=\alpha$, явлется равномерно наиболее мощным, т. $е$.

$$
\begin{array}{ll}
\inf _{W \in N_{\alpha}} \alpha(\mathbf{T}, \mathbf{u}, v)=\alpha\left(\mathbf{T}_{0}, \mathbf{u}_{0}, v\right), & v \leqslant r ; \\
\sup _{W \in N_{\alpha}} \beta(\mathbf{T}, \mathbf{u}, v)=\beta\left(\mathbf{T}_{0}, \mathbf{u}_{0}, v\right), & v>r .
\end{array}
$$

Д ока зат ел ь т в о. Обозначив через $c_{n}$ нормирующую постоянную $n$-мерного нормального закона, перепишем $P(W(\mathbf{T}, \mathbf{u}) \mid \theta)$ в интегральном виде:

$$
P(W(\mathbf{T}, \mathbf{u}) \mid \theta)=\underset{(\mathbf{T} \mathbf{y}+\mathbf{u})^{\mathrm{T}}}{c_{\mathbf{A}(\mathbf{T} \mathbf{y}+\mathbf{u}) \geq \mathbf{1}}} \exp _{\mathbf{T}}\left[-(\mathbf{y}-\mathbf{X} \theta)^{\mathrm{T}}(\mathbf{y}-\mathbf{X} \theta) / 2\right] d \mathbf{y} .
$$

Вводя новые обозначения $\mathbf{G}=\left(\mathbf{X}^{\mathrm{T}} \mathbf{X}\right)^{1 / 2} \mathbf{T}$ и $\left(\mathbf{X}^{\mathrm{T}} \mathbf{X}\right)^{1 / 2}\left(\theta-\theta_{0}\right)=\eta$, получим

$$
\begin{aligned}
& \alpha(\mathbf{T}, \mathbf{u}, v)=\sup _{\eta^{\mathrm{T}}{ } c_{n} \int v^{2}} c_{B} \exp \left(-\mathbf{e}^{\mathrm{T}} \mathbf{e} / 2\right) d \mathbf{e}, \\
& \beta(\mathbf{T}, \mathbf{u}, v)=\inf _{\eta^{\mathrm{T}}{ }_{\eta=v^{2}} c_{B} \int \exp \left(-\mathbf{e}^{\mathrm{T}} \mathbf{e} / 2\right) d \mathbf{e},}
\end{aligned}
$$

где $\quad B=\left\{\mathrm{e} \in R^{n}:\left[\mathbf{G e}+\mathbf{G X}\left(\mathbf{X}^{\mathrm{T}} \mathbf{X}\right)^{-1 / 2} \eta+\mathbf{T X} \theta_{0}\right]^{\mathrm{T}}\left[\mathbf{G e}+\mathbf{G X}\left(\mathbf{X}^{\mathrm{T}} \mathbf{X}\right)^{-1 / 2} \eta+\right.\right.$

$$
\left.\left.+\mathrm{TX} \theta_{0}\right] \geqslant 1\right\} \text {. }
$$

Из последних равенств в силу леммы $1\left[{ }^{4}\right]$ нетрудно видеть, что

$$
\begin{array}{r}
\inf _{\mathbf{u} \in R^{m}} \alpha(\mathbf{T}, \mathbf{u}, v) \geqslant \alpha\left(\mathbf{T},-\mathbf{T X} \theta_{0}, v\right), \\
\beta(\mathbf{T}, \mathbf{u}, v) \leqslant \beta\left(\mathbf{T},-\mathbf{T X} \theta_{0}, v\right),
\end{array}
$$

откуда следует, что $\mathbf{u}_{0}=-\mathbf{T X} \theta_{0}$ является оптимальным значением вектора и.

Существуют $\left[{ }^{5}\right]$ ортогональные матрицы $\mathbf{C}_{1}, \mathbf{C}_{2}$ порядка $(n \times n)$ и $\mathbf{B}_{1}, \mathbf{B}_{2}$ порядка $(m \times m)$ такие, что $\mathbf{G}=\mathbf{B}_{1}\left(\mathbf{H} \vdots \mathbf{O}_{m, n-m}\right) \mathbf{C}_{1}$ и $\left(\mathbf{X}^{\mathrm{T}} \mathbf{X}\right)^{-1 / 2} \mathbf{X}^{\mathrm{T}}=\mathbf{B}_{2}\left(\mathbf{I}_{m}: \mathbf{O}_{m, n-m}\right) \mathbf{C}_{2}$, где $\mathbf{H}-$ диагональная матрица с диагональными элементами $0<h_{1} \leqslant h_{2} \leqslant \ldots \leqslant h_{m}, \mathbf{I}_{m}-$ единичная матрица порядка $(m \times m), \quad \mathbf{O}_{m, n-m}-$ нулевая матрица порядка $m \times(n-m)$. Обозначив $\mathbf{C}=\mathbf{C}_{1} \mathbf{C}_{2}{ }^{\mathrm{T}}$, преобразуем (4) к виду

$$
\begin{aligned}
& \alpha\left(\mathbf{T}, \mathbf{u}_{0}, v\right)=\sup _{\eta^{\mathrm{T}} \eta=v^{2}} P_{\eta}(\mathbf{H}, \mathbf{N}), \\
& \beta\left(\mathbf{T}, \mathbf{u}_{0}, v\right)=\inf _{\eta^{\mathrm{T}}{ }_{\eta=v^{2}}} P_{\eta}(\mathbf{H}, \mathbf{N}),
\end{aligned}
$$

где $P_{\eta}(\mathbf{H}, \mathbf{N})=c_{m} \underset{(\mathbf{z}+\mathbf{N} \eta)^{\mathrm{T}}}{\mathbf{H}^{2}(\mathbf{z}+\mathbf{N} \eta) \geqslant 1} \exp _{1}\left(-\mathbf{z}^{\mathrm{T}} \mathbf{z} / 2\right) d \mathbf{z}, \mathbf{z}-m$-мерный вектор, $\mathbf{N}=\left(\mathbf{I}_{m}: \mathbf{O}_{m, n-m}\right) \mathbf{C}\left(\mathbf{I}_{m} \vdots \mathbf{O}_{m, n-m}\right)^{\mathrm{T}}$, Пусть вектор $\eta_{0}$ таков, что $\quad \eta_{0}{ }^{\mathrm{T}} \eta_{0}=r^{2}$ 
и $\mathbf{N} \eta_{0}=(\xi, 0, \ldots, 0)^{\mathrm{T}}, \quad \xi<0$. Тогда по лемме $1 *$ получаем

$$
\begin{array}{ll}
P_{s \eta_{0}}(\mathbf{H}, \mathbf{N})<P_{s \eta_{0}}\left(h \mathbf{I}_{m}, \mathbf{N}\right), & s>1, \\
P_{s \eta_{0}}(\mathbf{H}, \mathbf{N})>P_{s \eta_{0}}\left(h \mathbf{I}_{m}, \mathbf{N}\right), & 0<s<1,
\end{array}
$$

где $h>0$ выбрано так, что $P_{\eta_{0}}(\mathbf{H}, \mathbf{N})=P_{\eta_{0}}\left(h \mathbf{I}_{m}, \mathbf{N}\right)$. Кроме того, в силу леммы 2 при $h_{1}>h_{2}>0$ и $c>1$ имеем

$$
\begin{array}{ll}
P_{s \eta_{0}}\left(h_{1} \mathbf{I}_{m}, \mathbf{N}\right)<P_{s \eta_{0}}\left(h_{2} \mathbf{I}_{m}, c \mathbf{N}\right), & s>1, \\
P_{s \eta_{0}}\left(h_{1} \mathbf{I}_{m}, \mathbf{N}\right)>P_{s \eta_{0}}\left(h_{2} \mathbf{I}_{m}, c \mathbf{N}\right), & 0<s<1,
\end{array}
$$

где $h_{1}, h_{2}$ и с связаны условием

$$
P_{\eta_{0}}\left(h_{1} \mathbf{I}_{m}, \mathbf{N}\right)=P_{\eta_{0}}\left(h_{2} \mathbf{I}_{m}, c \mathbf{N}\right) .
$$

Заметим, что

$$
\eta_{0}^{\mathrm{T}} \mathbf{N}^{\mathrm{T}} \mathbf{N} \eta_{0}=\eta_{0}^{\mathrm{T}} \mathbf{C}^{* \mathrm{~T}} \mathbf{C}^{*} \eta_{0} \leqslant \eta_{0}^{\mathrm{T}} \eta_{0}
$$

где $\mathbf{C}^{*}$ - квадратная матрица порядка $(m \times m)$, занимающая верхний левый угол в ортогональной матрице С. Но неравенства (5) свидетельствуют о том, что увеличение нормы вектора $\mathbf{N} \eta_{0}$ улучшает качество критерия и, следовательно, для достижения равенства в (6) необходимо, чтобы $\mathbf{C}^{*}:=\mathbf{I}_{m}$.

Окончательно, выбрав $\mathbf{C}=\mathbf{I}_{n}$, т. е. $\mathbf{C}_{1}=\mathbf{C}_{2}$, и скаляр $h$ таким, что $P_{\eta_{0}}\left(h \mathbf{I}_{m}, \mathbf{I}_{m}\right)=\alpha$, мы построили в силу неравенств (5) равномерно наиболее мощный критерий в смысле (3).

Т е о р е м а 2. Пусть $\mathrm{A}_{2}=\mathrm{pp}^{\mathrm{T}}, \mathrm{p}-$ известный m-мерный вектор. Тогда в классе $\mathbf{N}_{\alpha}$ критерий $W_{1}$, которому соответствуют $\mathbf{T}_{1}=t_{1}\left(\mathbf{X}^{\mathrm{T}} \mathbf{X}\right)^{-1} \mathbf{X}^{\mathrm{T}} u$ $\mathbf{u}_{1}=-\mathbf{T}_{1} \mathbf{X} \theta_{0}$, где $t_{1}$ выбирается из условия $\gamma\left(\mathbf{T}_{1}, \mathbf{u}_{1}, r\right)=\alpha$, является равномерно наиболее моцным в смысле (3).

Д оказ ательст в о. Так же, как и в теореме 1, можно показать, что для оптимального критерия $W_{1}$ необходимо, чтобы $\mathrm{p}^{\mathrm{T}} \mathrm{T}_{1}^{\mathrm{T}} \mathbf{X} \theta_{0}+$ $+\mathrm{p}^{\mathrm{T}} \mathbf{u}=0$. Тогда, вводя обозначения $\mathrm{p}^{\mathrm{T}} \mathrm{T}=\mathrm{s}^{\mathrm{T}} \in R^{n} \quad$ и $\quad \theta-\theta_{0}=$ $=\eta \in R^{m}$, получаем

$$
\begin{aligned}
& \alpha\left(\mathbf{T}, \mathbf{u}_{0}, v\right)=\sup _{\left(\eta^{\mathrm{T}} \mathbf{p}\right)^{2}=v^{2}} c_{n} \int_{\left(\mathbf{s}^{\mathrm{T}} \mathbf{e}+\mathbf{s}^{\mathrm{T}} \mathrm{T} \eta\right)^{2} \geqslant 1} \exp \left(-\mathrm{e}^{\mathrm{T}} \mathbf{e} / 2\right) d \mathbf{e}, \\
& \beta\left(\mathbf{T}, \mathbf{u}_{0}, v\right)=\inf c_{n} \quad \int_{\mathrm{T}} \exp \left(-\mathbf{e}^{\mathrm{T}} \mathbf{e} / 2\right) d \mathbf{e} . \\
& \left(\eta^{\mathrm{T}} \mathrm{p}\right)^{2}=v^{2} \quad\left(\mathrm{~s}^{\mathrm{T}} \mathrm{e}+\mathrm{s}^{\mathrm{T}} \mathrm{T} \eta\right)^{2} \geqslant 1
\end{aligned}
$$

Сделав замену переменных $\mathbf{B e}=\mathbf{z}=\left(z_{1}, z_{2}, \ldots, z_{n}\right)^{\mathrm{T}}$, где $\mathbf{B}-$ ортогональная матрица, первая строка которой $\mathbf{s}^{\mathrm{T}} / \sqrt{\mathbf{s}^{\mathrm{T}} \mathbf{s}}$, преобразуем функции $\alpha(\mathbf{T}, \mathbf{u}, v)$ и $\beta(\mathbf{T}, \mathbf{u}, v)$ к виду

$$
\begin{aligned}
& \alpha(\mathbf{T}, \mathbf{u}, v)=\sup _{\left(\eta^{\mathrm{T}} \mathrm{p}\right)^{2}=v^{2}} c_{1} \quad \int \quad \exp \left(-z_{1}^{2} / 2\right) d z_{1}, \\
& \left(z_{1} \sqrt{s^{T} s+s^{T}} x \eta\right)^{2} \geqslant 1 \\
& \beta(\mathbf{T}, \mathbf{u}, v)=\inf _{\left(\eta^{\mathrm{T}} \mathbf{p}\right)^{2}=v^{2}} c_{1} \underset{\left(z_{1} \sqrt{\mathbf{s}^{\mathrm{T}} \mathrm{s}+\mathbf{s}^{\mathrm{T}}} \mathbf{x} \eta\right)^{2} \geqslant 1}{\int} \exp \left(-z_{1}^{2} / 2\right) d z_{1} .
\end{aligned}
$$

* Формулировка и доказательство лемм приведены в конце статьи, 
Заметим, что существует скаляр $\gamma \in R^{1}$ такой, что вектор $\mathbf{s}_{0}=\mathbf{T}_{0}{ }^{\mathrm{T}} \mathbf{p}$ удовлетворяет уравнению

$$
\mathbf{s}_{0}^{\mathrm{T}} \mathbf{X}=\gamma \mathbf{p}^{\mathrm{T}},
$$

в противном случае $\sup _{\mathrm{T}^{2}}\left(\mathbf{s}_{0}^{\mathrm{T}} \mathbf{X} \eta\right)=\infty \quad$ и $\alpha\left(\mathbf{T}, \mathbf{u}_{0}, v\right)=1$, что проти$\left.(\eta)^{\mathrm{T}}\right)^{2}=v^{2}$

воречит условию $W \in N_{\alpha}$. Общий вид решения уравнения (7) относительно $\mathrm{s}_{0}$ принимает вид $\left[{ }^{5}\right]$

$$
\mathbf{s}_{0}=\gamma\left[\mathbf{X}\left(\mathbf{X}^{\mathrm{T}} \mathbf{X}\right)^{-1} \mathbf{p}+\left(\mathbf{I}_{n}-\left(\mathbf{X}\left(\mathbf{X}^{\mathrm{T}} \mathbf{X}\right)^{-1} \mathbf{X}^{\mathrm{T}}\right) \mathbf{L}\right],\right.
$$

где $\mathbf{L}-$ произвольный вектор из $R^{n}$. Используя обозначения теоремы 1 , получаем

$$
P_{\eta}(\mathbf{H}, \mathbf{N})=P_{1}\left(\mathbf{s}_{0}^{\mathrm{T}} \mathbf{s}_{0}, \mathbf{s}_{0}^{\mathrm{T}} \mathbf{X} \mathbf{p} / \sqrt{\left.\mathbf{s}_{0}^{\mathrm{T}} \mathbf{s}_{0}\right)},\right.
$$

где

$$
\mathbf{s}_{0}^{\mathrm{T}} \mathbf{s}_{0}=\gamma^{2}\left[\mathbf{p}^{\mathrm{T}}\left(\mathbf{X}^{\mathrm{T}} \mathbf{X}\right)^{-1} \mathbf{p}+\mathbf{L}^{\mathrm{T}}\left(\mathbf{I}_{n}-\mathbf{X}\left(\mathbf{X}^{\mathrm{T}} \mathbf{X}\right)^{-1} \mathbf{X}^{\mathrm{T}}\right) \mathbf{L}\right]
$$

и

$$
\mathbf{s}_{0}^{\mathrm{T}} \mathbf{X} \mathbf{p}=\gamma \eta+(\gamma-1) \mathbf{L}^{\mathrm{T}} \mathbf{X} \eta .
$$

Из леммы 2 следует, что для оптимального критерия надо взять $\mathbf{L}=\mathbf{O} \in R^{m}$, что и доказывает теорему.

Л ем м а 1. Пусть $h_{2}>h_{1}, a \mathbf{H}_{1}-$ диагональная матрица порядка $(m \times m)$ с диагональными элементами $h, h, h_{3}, \ldots, h_{m}$, где $h$ выбрано так, что $P_{\eta_{0}}(\mathbf{H}, \mathbf{N})=P_{\eta_{0}}\left(\mathbf{H}_{1}, \mathbf{N}\right)$. Тогда

$$
\begin{array}{ll}
P_{s \eta_{0}}(\mathbf{H}, \mathbf{N})<P_{s \eta_{0}}\left(\mathbf{H}_{1}, \mathbf{N}\right), & s>1, \\
P_{s \eta_{0}}(\mathbf{H}, \mathbf{N})>P_{s \eta_{0}}\left(\mathbf{H}_{1}, \mathbf{N}\right), & 0<s<1 .
\end{array}
$$

Док а з а тел ьство. Достаточно показать, что из равенства $P_{s_{0} \eta_{0}}(\mathbf{H}, \mathbf{N})=P_{s_{0} \eta_{0}}\left(\mathbf{H}_{1}, \mathbf{N}\right)$ для некоторого $s_{0}>0$ следует

$$
\left[d\left(P_{s \eta_{0}}(\mathbf{H}, \mathbf{N}) / d s\right] / s=s_{0}<\left[d\left(P_{s \eta_{0}}\left(\mathbf{H}_{1}, \mathbf{N}\right) / d s\right] / s=s_{0} .\right.\right.
$$

По определению

$$
\begin{gathered}
{\left[d\left(P_{s \eta_{0}}(\mathbf{H}, \mathbf{N}) / d s\right] / s_{s=s_{0}}=-c_{m} \xi \int_{z \in J\left(\mathbf{H}^{2}\right)} \exp \left(-\sum_{i=2}^{m} z_{i}^{2} / 2-\right.\right.} \\
\left.-\left(z_{1}-s_{0} \xi\right)^{2} / 2\right)\left(z_{1}-s_{0} \xi\right) \mathrm{d} \mathbf{z},
\end{gathered}
$$

где $J\left(\mathbf{H}^{2}\right)=\left\{\mathbf{z} \in R^{m}: \mathbf{z}^{\mathrm{T}} \mathbf{H}^{2} \mathbf{z} \leqslant 1\right\}$. Из (9) с помощью несложного преобразования получаем

$$
\begin{gathered}
{\left[d\left(P_{s \eta_{0}}(\mathbf{H}, \mathbf{N}) / d s\right] / s=s_{0}^{\prime}=s_{0} \xi^{2} P_{s_{0} \eta_{0}}(\mathbf{H}, \mathbf{N})-c_{m} \xi \times\right.} \\
\times \int_{\mathrm{z} \in J\left(\mathbf{H}^{2}\right) \cap\left\{\mathbf{z} \in R^{m: z_{1}} \geqslant 0\right\}} \exp \left(-\sum_{i=2}^{m} z_{i}^{2} / 2\right)\left[\exp \left(-\left(s_{0} \xi-z_{1}\right)^{2} / 2\right)+\exp \left(-\left(s_{0} \xi+z_{1}\right)^{2} / 2\right)\right] \times \\
\times z_{1} \operatorname{th}\left(s_{0} \xi z_{1}\right) d z_{1} d z_{2} \ldots d z_{m},
\end{gathered}
$$

где th $(v)=\left(e^{v}-e^{-v}\right) /\left(e^{-v}+e^{v}\right), \quad v \in R^{1}$. Выписывая аналогичным образом $\left[d\left(P_{s \eta_{0}}\left(\mathbf{H}_{1}, \mathbf{N}\right) / d s\right] /_{s=s_{0}}\right.$, находим 


$$
\begin{gathered}
c_{m}^{-1}\left[d\left(P_{s \eta_{0}}(\mathbf{H}, \mathbf{N})-P_{s \eta_{0}}\left(\mathbf{H}_{1}, \mathbf{N}\right)\right) / d s\right] / s=s_{0}=\int_{z \in F_{0} \backslash F_{1}} \exp \left(-\sum_{i=2}^{m} z_{i}^{2} / 2\right) \times \\
\times\left[\exp \left(-\left(s_{0} \xi-z_{1}\right)^{2} / 2\right)+\exp \left(-\left(s_{0} \xi+z_{1}\right)^{2} / 2\right)\right] \times \\
\times z_{1} \operatorname{th}\left(s_{0} \xi z_{1}\right) d \mathbf{z}-\int_{\mathbf{z} \in F_{1} \backslash F_{0}} \exp \left(-\sum_{i=2}^{m} z_{i}^{2} / 2\right)\left[\exp \left(-\left(s_{0} \xi-z_{1}\right)^{2} / 2\right)+\right. \\
\left.+\exp \left(-\left(\mathrm{s}_{0} \xi+z_{1}\right)^{2} / 2\right)\right] z_{1} \operatorname{th}\left(s_{0} \xi z_{1}\right) d z,
\end{gathered}
$$

где

$$
\begin{aligned}
& F_{0}=J\left(\mathbf{H}^{2}\right) \cap\left\{\mathrm{z} \in R^{m}: z_{1} \geqslant 0\right\}, \\
& F_{1}=J\left(\mathbf{H}_{1}^{2}\right) \cap\left\{\mathrm{z} \in R^{m}: z_{1} \geqslant 0\right\} .
\end{aligned}
$$

Очевидно, что если $\left(z_{1}^{\prime}, z_{2}^{\prime}, \ldots, z_{m}^{\prime}\right) \in F_{0} \backslash F_{1}$, а $\left(z_{1}^{\prime \prime}, z_{2}^{\prime \prime}, \ldots, z_{m}^{\prime \prime}\right) \in$ $\in F_{1} \backslash F_{0}$, то $z_{1}^{\prime}>z_{1}^{\prime \prime}$. Но функция $z_{1}$ th $\left(s_{0} \xi z_{1}\right)$ убывает по $z_{1}>0$, следовательно, выражение (10) не положительно, что и доказывает лемму 1 .

Л е м м а 2. Пусть $P(t, \eta)=\int_{\mathbf{z} \in \bar{K}(t, \eta)} \exp \left(-\mathbf{z}^{\mathrm{T}} \mathbf{z} / 2\right) d \mathbf{z}$, где

$$
\bar{K}(t, \eta)=\left\{\mathbf{z} \in R^{m}: t^{2}(\mathbf{z}+\eta)^{\mathrm{T}}(\mathbf{z}+\eta) \geqslant 1\right\}, \quad t \neq 0, \eta \in R^{m} .
$$

Выберем $\eta_{0}=\left(h_{0}, 0, \ldots, 0\right) \in R^{m}, \quad \eta_{1}=\left(h_{1}, 0, \ldots, 0\right) \in R^{m}$, $0>h_{1}>h_{0} u 0<t_{0}<t_{1}$ так, что $P\left(t_{0}, \eta_{0}\right)=P\left(t_{1}, \eta_{1}\right)$. Тогда

$$
\begin{array}{ll}
P\left(t_{0}, s \eta_{0}\right)>P\left(t_{1}, s \eta_{1}\right), & s>1, \\
P\left(t_{0}, s \eta_{0}\right)<P\left(t_{1}, s \eta_{1}\right), & 0<s<1 .
\end{array}
$$

Д ок а з тельство. Достаточно показать, что из равенства $P\left(t_{0}, s_{0} \eta_{0}\right)=P\left(t_{1}, s_{0} \eta_{1}\right)$ для некоторого $s_{0}>0$ следует

$$
\left[d\left(P\left(t_{0}, s \eta_{0}\right)\right) / d s\right] / s=s_{0}>\left[d\left(P\left(t_{1}, s \eta_{1}\right)\right) / d s\right] / s=s_{1} .
$$

По определению $P(t, \eta)$ для $i=0,1$ имеем

$$
\left[d\left(P\left(t_{i}, s \eta_{i}\right)\right) / d s\right] / s=s_{0}=-h_{i} \int_{\mathbf{z} \in M_{t}} \exp \left(-\mathbf{z}^{\mathrm{T}} \mathbf{z} / 2\right) z_{1} d \mathbf{z}
$$

где $M_{i}=\left\{\mathrm{z} \in R^{m}: t_{i}{ }^{2}\left(\mathrm{z}+s_{0} \eta_{i}\right)^{\mathrm{T}}\left(\mathrm{z}+s_{0} \eta_{i}\right) \leqslant 1\right\}$. Используя разложение $M_{i}=\left\{M_{i} \backslash\left(M_{1-i} \cap M_{i}\right)\right\} \bigcup\left\{M_{i} \cap M_{1-i}\right\}$ и формулу (12), получаем

$$
\begin{aligned}
& {\left[d\left(P\left(t_{1}, s \eta_{1}\right)-P\left(t_{0}, s \eta_{0}\right)\right) / d s\right]_{s=s_{0}}=\left(h_{0}-h_{1}\right) \int_{\mathbf{z} \in M_{0} \cap M_{1}} \exp \left(-\mathbf{z}^{\mathrm{T}} \mathbf{z} / 2\right) z_{1} d \mathbf{z}+} \\
& \left.+h_{0} \int_{\mathbf{z} \in M_{0} \backslash\left(M_{\circ} \cap M_{1}\right)} \exp \left(-\mathbf{z}^{\mathrm{T}} \mathbf{z} / 2\right) z_{1} d \mathbf{z}-h_{1} \underset{\left.z \in M_{1}\right\}\left(M_{0} \cap M_{1}\right)}{\int} \exp \left(-\mathbf{z}^{\mathrm{T}} \mathbf{z}\right) / 2\right) z_{1} d \mathbf{z} .
\end{aligned}
$$

Поскольку $h_{1}>h_{0}$ и $P\left(t_{0}, s_{0} \eta_{0}\right)=P\left(t_{1}, s_{0} \eta_{1}\right)$, то если $\left(z_{1}^{\prime}, z_{2}^{\prime}, \ldots\right.$, $\left.z_{m}^{\prime}\right) \in M_{0} \backslash\left\{M_{0} \cap M_{1}\right\}$, a $\left(z_{1}^{\prime \prime}, z_{2}^{\prime \prime}, \ldots, z_{m}^{\prime \prime}\right) \in M_{1} \backslash\left\{M_{0} \cap M_{1}\right\}$, то, очевидно, $z_{1}^{\prime}>z_{1}^{\prime \prime}$. Используя этот факт и теорему о среднем, легко получаем, что первое слагаемое в (13), а также разность двух других членов в (13)отрицательны, что и доказывает лемму 2 , 


\section{Л ИТ Е РАТ У РА}

1. B u nke, O., Math. Operationsforsch. u. Statist., 6, № 5, 697-701 (1975).

2. К ук с Я., О льм ан В., Изв. АН ЭССР, Физ. Матем., 21, № 1, 66-72 (1972).

3. К укс Я., О льм а н В., Изв. АН ЭССР, Физ. Матем., 20, № 4, 480-482 (1971).

4. О ль м а н В., Изв. АН ЭССР, Физ. Матем., 23, № 2, 127-134 (11974).

5. Р а о С. Р., Линейные статистические методы и их применения, М., «Наука», 1968.

Институт кибернетики

Академии наук Эстонской ССР
Поступила в редакцию 22/XII 1978

V. OLMAN

\section{LINEAARSE MUDELI PARAMEETRI ANTUD ELLIPSOIDI KUULUMISE HOPOTEESI KONTROLL}

On kontrollitud lineaarse regressioonimudeli parameetri $\theta$ kohta püstitatud hüpoteesi $H_{0}:\left(\theta-\theta_{0}\right)^{\mathrm{T}} \mathbf{A}\left(\theta-\theta_{0}\right) \leqslant r^{2}$ öigsust. Maatriksi A kahe erikuju korral on leitud lineaarsete statistikutega tsentreeritavate ellipsoidide hulgast kôige suurema võimsusega kriitiline piirkond.

\section{OLMAN}

\section{TESTING OF THE HYPOTHESIS OF LINEAR REGRESSION PARAMETER BELONGING TO FIXED ELLIPSOID}

Linear regression model $E \mathbf{Y}=\mathbf{X} \theta$ with independent errors in observations is considered. Statistical hypothesis $H_{0}:\left(\theta-\theta_{0}\right)^{\mathrm{T}} \mathbf{A}\left(\theta-\theta_{0}\right) \leqslant r^{2}$ is tested against alternative $K$ : $:\left(\theta-\theta_{0}\right)^{\mathrm{T}} \mathbf{A}\left(\theta-\theta_{0}\right)>r^{2}$, where matrix $\mathbf{A}$ and vector $\theta_{0}$ are fixed. The maximal risk over surface $\left(\theta-\theta_{0}\right)^{\mathrm{T}} \mathbf{A} \quad\left(\theta-\theta_{0}\right)=v^{2}$ is taken as error of first kind for $v \leqslant r$ and as error of second kind for $v>r$. The ellipsoids centered by linear statistics of observations are investigated as critical regions. For two special cases of matrix A the uniformly most powerful tests are constructed. 